\title{
On Regular Generalized Open Sets In Topological Space
}

\author{
S. Syed Ali Fathima \\ Assistant Professor Of Mathematics \\ Sadakathulla Appa College \\ Tirunelveli , Tamil Nadu, India- 627011
}

\author{
M. Mariasingam \\ Associate Professor Of Mathematics \\ V.O.Chidambaram College \\ Thoothukudi, Tamil Nadu, India -628 008
}

\begin{abstract}
In this paper we introduce and study \#regular generalized open (briefly, \#rg-open) sets in topological space and obtain some of their properties. Also, we introduce \#rgneighbourhood (shortly \#rg-nbhd) in topological spaces by using the notion of \#rg-open sets. Applying \#rg-closed sets we introduce \#rg-closure and discuss some basic properties of this.
\end{abstract}

\section{KEYWORDS}

rg-open sets,, rg-nbhd, rg-closure.

\section{AMS SUBJECT CLASSIFICATION (2000) 1. INTRODUCTION:}

Regular open sets and rw-open sets have been introduced and investigated by Stone[16] and Benchalli and Wali[1] respectively. Levine[8,9], Biswas[3], Cameron[4], Sundaram and Sheik john[17], Bhattacharyya and Lahiri[2], Nagaveni[12], Pushpalatha[15], Gnanambal[6], Gnanambal and Balachandran[7], Palaniappan and Rao[13] and Maki, Devi and Balachandran[10] introduced and investigated semi open sets, generalized closed sets, regular semi open sets, weakly closed sets, semi generalized closed sets, weakly generalized closed sets, strongly generalized closed sets, generalized pre-regular closed sets, regular generalized closed sets, and generalized $\alpha$-generalized closed sets respectively. We introduce a new class of sets called \#regular generalized open sets which is properly placed in between the class of open sets and the class of rg-open sets.

Throughout this paper $(\mathrm{X}, \tau)$ represents a topological space on which no separation axiom is assumed unless otherwise mentioned. For a subset A of a topological space X, $\operatorname{cl}(\mathrm{A})$ and int(A) denote the closure of $\mathrm{A}$ and the interior of $\mathrm{A}$ respectively. $\mathrm{X} \backslash \mathrm{A}$ or $\mathrm{A}^{\mathrm{c}}$ denotes the complement of $\mathrm{A}$ in $\mathrm{X}$. We recall the following definitions and results.

\subsection{Definition}

A subset $\mathrm{A}$ of a space $\mathrm{X}$ is called

1) a preopen set[11] if $\mathrm{A} \subseteq$ intcl (A) and a preclosed set if clint $(\mathrm{A}) \subseteq \mathrm{A}$.

2) a semiopen set[8] if $\mathrm{A} \subseteq$ clint $(\mathrm{A})$ and a semiclosed set if intcl $(\mathrm{A}) \subseteq \mathrm{A}$.

3) a regular open set[16]if $\mathrm{A}=\operatorname{intcl}(\mathrm{A})$ and a regular closed set if $\mathrm{A}=\operatorname{clint}(\mathrm{A})$.

4) a $\pi$ - open set[20] if $A$ is a finite union of regular open sets.

5) regular semi open[4]if there is a regular open $U$ such

$\mathrm{U} \subseteq \mathrm{A} \subseteq \mathrm{cl}(\mathrm{U})$.

\subsection{Definition}

A subset $\mathrm{A}$ of $(\mathrm{X}, \tau)$ is called

1) generalized closed set (briefly, g-closed)[9] if $\mathrm{cl}(\mathrm{A}) \subseteq \mathrm{U}$ whenever $\mathrm{A} \subseteq \mathrm{U}$ and $\mathrm{U}$ is open in $\mathrm{X}$.

2) regular generalized closed set (briefly, rg-closed)[13] if $\mathrm{cl}(\mathrm{A}) \subseteq \mathrm{U}$ whenever $\mathrm{A} \subseteq \mathrm{U}$ and $\mathrm{U}$ is regular open in $\mathrm{X}$.

3) generalized preregular closed set (briefly, gpr-closed)[6] if $\operatorname{pcl}(\mathrm{A}) \subseteq \mathrm{U}$ whenever $\mathrm{A} \subseteq \mathrm{U}$ and $\mathrm{U}$ is regular open in $\mathrm{X}$.

4) weakely generalized closed set (briefly, wg-closed)[12] if clint $(\mathrm{A}) \subseteq \mathrm{U}$ whenever $\mathrm{A} \subseteq \mathrm{U}$ and $\mathrm{U}$ is open in $\mathrm{X}$

5) $\pi$-generalized closed set (briefly, $\pi$ g-closed)[5] if $\mathrm{cl}(\mathrm{A}) \subseteq \mathrm{U}$ whenever $\mathrm{A} \subseteq \mathrm{U}$ and $\mathrm{U}$ is $\pi$-open in $\mathrm{X}$.

6) weakely closed set (briefly, w-closed)[15] if $\operatorname{cl}(\mathrm{A}) \subseteq \mathrm{U}$ whenever $\mathrm{A} \subseteq \mathrm{U}$ and $\mathrm{U}$ is semi open in $\mathrm{X}$.

7) regular weakly generalized closed set (briefly, rwgclosed)[12] if $\operatorname{clint}(\mathrm{A}) \subseteq \mathrm{U}$ whenever $\mathrm{A} \subseteq \mathrm{U}$ and $\mathrm{U}$ is regular open in X.

8) rw-closed [1] if $\operatorname{cl}(\mathrm{A}) \subseteq \mathrm{U}$ whenever $\mathrm{A} \subseteq \mathrm{U}$ and $\mathrm{U}$ is regular semi open.

9) $*$ g-closed [19] if $\operatorname{cl}(\mathrm{A}) \subseteq \mathrm{U}$ whenever $\mathrm{A} \subseteq \mathrm{U}$ and $\mathrm{U}$ is wopen.

10)\#rg-closed[18] if $\operatorname{cl}(\mathrm{A}) \subseteq \mathrm{U}$ whenever $\mathrm{A} \subseteq \mathrm{U}$ and $\mathrm{U}$ is rwopen.

\section{2. \#REGULAR GENERALIZED OPEN SETS AND \#REGULAR GENERALIZED NEIGHBOURHOODS.}

\subsection{Definition}

A subset $\mathrm{A}$ of a space $\mathrm{X}$ is called \#regular generalized open (briefly \#rg-open) set if its complement is \#rg-closed. The family of all \#rg- open sets in $\mathrm{X}$ is denoted by \#RGO(X).

\subsection{Remark}

$\operatorname{cl}(\mathrm{X} \backslash \mathrm{A})=\mathrm{X} \backslash \mathrm{int}(\mathrm{A})$.

\subsection{Theorem}

A subset $\mathrm{A}$ of $\mathrm{X}$ is \#rg-open if and only if $\mathrm{F} \subseteq \operatorname{int}(\mathrm{A})$ whenever $\mathrm{F}$ is rw-closed and $\mathrm{F} \subseteq \mathrm{A}$. 


\section{Proof}

(Necessity). Let $\mathrm{A}$ be \#rg-open . Let $\mathrm{F}$ be rw-closed and $\mathrm{F} \subseteq \mathrm{A}$ then $X \backslash A \subseteq X \backslash F$, whenever $X \backslash F$ is rw-open. Since $X \backslash A$ is \#rgclosed, $\operatorname{cl}(\mathrm{X} \backslash \mathrm{A}) \subseteq \mathrm{X} \backslash \mathrm{F}$. By Remark 2.2, $\mathrm{X} \backslash \operatorname{int}(\mathrm{A}) \subseteq \mathrm{X} \backslash \mathrm{F}$. That is $\mathrm{F} \subseteq \operatorname{int}(\mathrm{A})$.

(Sufficiency). Suppose $\mathrm{F}$ is rw-closed and $\mathrm{F} \subseteq \mathrm{A}$ implies $F \subseteq \operatorname{int}(A)$. Let $X \backslash A \subseteq U$ where $U$ is rw-open. Then $X \backslash U \subseteq A$, where $X \backslash U$ is rw-closed. By hypothesis $X \backslash U \subseteq \operatorname{int}(A)$. That is $\mathrm{X} \backslash$ int $(\mathrm{A}) \subseteq \mathrm{U}$. By remark2.2 $\mathrm{cl}(\mathrm{X} \backslash \mathrm{A}) \subseteq \mathrm{U}$, implies, $\mathrm{X} \backslash \mathrm{A}$ is \#rgclosed and $\mathrm{A}$ is \#rg-open.

\subsection{Theorem}

If $\operatorname{int}(\mathrm{A}) \subseteq \mathrm{B} \subseteq \mathrm{A}$ and $\mathrm{A}$ is \#rg-open, then $\mathrm{B}$ is \#rg-open.

\section{Proof}

Let $\mathrm{A}$ be \#rg-open set and $\operatorname{int}(\mathrm{A}) \subseteq \mathrm{B} \subseteq \mathrm{A}$. Now $\operatorname{int}(\mathrm{A}) \subseteq \mathrm{B} \subseteq \mathrm{A}$ implies $\mathrm{X} \backslash \mathrm{A} \subseteq \mathrm{X} \backslash \mathrm{B} \subseteq \mathrm{X}$ int $(\mathrm{A})$. That is $\mathrm{X} \backslash \mathrm{A} \subseteq \mathrm{X} \backslash \mathrm{B} \subseteq$ $\mathrm{cl}(\mathrm{X} \backslash \mathrm{A})$. Since $\mathrm{X} \backslash \mathrm{A}$ is \#rg-closed, $\mathrm{X} \backslash \mathrm{B}$ is \#rg-closed and $\mathrm{B}$ is \#rg-open.

\subsection{Remark}

For any $\mathrm{A} \subseteq \mathrm{X}, \operatorname{int}(\mathrm{cl}(\mathrm{A}) \backslash \mathrm{A})=\phi$.

\subsection{Theorem}

If $\mathrm{A} \subseteq \mathrm{X}$ is $\#$ rg-closed then $\operatorname{cl}(\mathrm{A}) \backslash \mathrm{A}$ is $\#$ rg-open.

\section{Proof}

Let $\mathrm{A}$ be \#rg-closed. Let $\mathrm{F}$ be rw-closed set such that $\mathrm{F} \subseteq \mathrm{cl}(\mathrm{A}) \backslash \mathrm{A}$. Then by theorem.2.7 [18], $\mathrm{F}=\phi$. So, $\mathrm{F} \subseteq$ $\operatorname{int}(\mathrm{cl}(\mathrm{A}) \backslash \mathrm{A})$. This shows $\mathrm{cl}(\mathrm{A}) \backslash \mathrm{A}$ is \#rg-open.

\subsection{Theorem}

Every open set in $\mathrm{X}$ is \#rg-open but not conversely.

\section{Proof}

Let $\mathrm{A}$ be an open set in a space X. Then X\A is closed set. By theorem 2.1[18], $\mathrm{X} \backslash \mathrm{A}$ is \#rg-closed. Therefore $\mathrm{A}$ is \#rg-open set in X.

The converse of the theorem need not be true, as seen from the following example.

\subsection{Example}

Let $X=\{a, b, c, d\}$ be with topology $\tau=\{X, \phi,\{a\},\{b\},\{a . b\}$, $\{a . b, c\}\}$ then the set $A=\{b, c\}$ is \#rg-open but not open set in $\mathrm{X}$.

\subsection{Corollary}

Every regular open set is \#rg - open but not conversely.

\section{Proof}

Follows from Stone [16] and theorem 2.7.

\subsection{Corollary}

Every $\pi$-open set is \#rg-open but not conversely.

\section{Proof}

Follows from Dontchev and Noiri [5] and theorem 2.7.

\subsection{Theorem}

Every \#rg-open sets in $\mathrm{X}$ is $\mathrm{rg}$-open set in $\mathrm{X}$, but not conversely..

\section{Proof}

Let A be \#rg-open set in space $\mathrm{X}$. Then $\mathrm{X} \backslash \mathrm{A}$ is \# rg-closed set in X. By theorem 2.2[18], X\A is rg-closed set in X. Therefore A is rg-open in $\mathrm{X}$.

The converse of the above theorem need not be true as seen from the following example.

\subsection{Example}

Let $X=\{a, b, c, d\}$ be with topology $\tau=\{X, \phi,\{a\},\{b\},\{a . b\}$, $\{a . b, c\}\}$ then the set $A=\{c, d\}$ is rg-open but not \#rg-open set in $\mathrm{X}$.

\subsection{Theorem}

Every \#rg-open set in $\mathrm{X}$ is $* \mathrm{~g}$-open set in $\mathrm{X}$, but not conversely.

\section{Proof}

Let A be \#rg-open set in space X. Then X\A is \# rg-closed set in $\mathrm{X}$. By theorem 2.3[18], $\mathrm{X \backslash A}$ is $*$ g-closed set in $\mathrm{X}$. Therefore $\mathrm{A}$ is $* \mathrm{~g}$-open in $\mathrm{X}$.

The converse of the above theorem need not be true as seen from the following example

\subsection{Example}

Let $X=\{a, b, c, d\}$ be with topology $\tau=\{X, \phi,\{a\},\{b\},\{a . b\}\}$ then the set $A=\{a, b, d\}$ is *g-open but not \#rg-open set in $X$.

\subsection{Theorem}

Every \#rg-open set in $\mathrm{X}$ is g-open, but not conversely.

\section{Proof.}

Let $\mathrm{A}$ be \#rg-open set in $\mathrm{X}$. Then $\mathrm{A}^{\mathrm{c}}$ is \#rg-closed set in $\mathrm{X}$. By theorem 2.5[18], $\mathrm{A}^{\mathrm{c}}$ is g-closed set in X. Hence $\mathrm{A}$ is g-open in $\mathrm{X}$.

The converse of the above theorem need not be true as seen from the following example.

\subsection{Example.}

Let $X=\{a, b, c, d\}$ be with topology $\tau=\{X, \phi,\{a\},\{b\},\{a . b\}\}$ then the set $A=\{a, b, c\}$ is g-open but not open in $X$.

\subsection{Theorem}

If a subset $\mathrm{A}$ of a space $\mathrm{X}$ is \#rg-open then it is $\pi \mathrm{g}$-open set in $\mathrm{X}$

\section{Proof}

Let A be \#rg-open set in space X. Then X\A is \# rg-closed set in $\mathrm{X}$. By theorem 2.4[18], $\mathrm{X} \backslash \mathrm{A}$ is $\pi$ g-closed set in $\mathrm{X}$ Therefore $\mathrm{A}$ is $\pi \mathrm{g}$-open in $\mathrm{X}$.

The converse of the above theorem need not be true as seen from the following example.

\subsection{Example}

Let $X=\{a, b, c, d\}$ be with topology $\tau=\{X, \phi,\{a\},\{b\},\{a . b\}$, $\{a, b, c\}\}$ then the set $A=\{a, b, d\}$ is $\pi$ g-open but not \#rg-open set in X.

\subsection{Remark}

The following example shows that \#rg-open sets are independent of rw-open sets, semi open sets, $\alpha$-open sets, g $\alpha$ open sets, sg-open sets, semi pre open sets, pre open sets and swg-open sets.

\subsection{Example}

Let $X=\{a, b, c, d\}$ be with topology $\tau=\{\phi, X,\{a\},\{b\},\{a, b\}$, $\{a, b, c\}\}$. Then

(i) \#rg-open sets in $(\mathrm{X}, \tau)$ are $\phi, X,\{a\},\{b\},\{c\},\{a, b\},\{b, c\}$, $\{a, c\},\{a, b, c\}$.

(ii) rw-open sets in $(X, \tau)$ are $\phi, X,\{a\},\{b\},\{c\},\{d\},\{a, b\}$, $\{c, d\},\{a, b, c\}$.

(iii) semi open sets in $(X, \tau)$ are $\phi, X,\{a\},\{b\},\{a, b\},\{b, c\}$ $\{\mathrm{a}, \mathrm{d}\},\{\mathrm{b}, \mathrm{d}\},\{\mathrm{a}, \mathrm{c}\},\{\mathrm{b}, \mathrm{c}, \mathrm{d}\},\{\mathrm{a}, \mathrm{c}, \mathrm{d}\},\{\mathrm{a}, \mathrm{b}, \mathrm{d}\},\{\mathrm{a}, \mathrm{b}, \mathrm{c}\}$.

(iv) $\alpha$-open sets in $(\mathrm{X}, \tau)$ are $\phi, \mathrm{X},\{\mathrm{a}\},\{\mathrm{b}\},\{\mathrm{a}, \mathrm{b}\},\{\mathrm{a}, \mathrm{b}, \mathrm{d}\}$ $\{\mathrm{a}, \mathrm{b}, \mathrm{c}\}$ 
(v) ga-open sets in $(\mathrm{X}, \tau)$ are $\phi, \mathrm{X},\{\mathrm{a}\},\{\mathrm{b}\},\{\mathrm{a}, \mathrm{b}\},\{\mathrm{a}, \mathrm{b}, \mathrm{d}\}$, $\{a, b, c\}$.

(vi) sg- open sets in $(\mathrm{X}, \tau)$ are $\phi, X,\{a\},\{b\},\{a, b\},\{a, d\}$, $\{b, c\},\{a, c\},\{b, d\},\{a, c, d\},\{a, b, d\},\{a, b, c\},\{b, c, d\}$.

(vii) semi pre open sets in $(X, \tau)$ are $\phi, X,\{a\},\{b\},\{a, b\}$, $\{a, d\},\{b, c\},\{a, c\},\{b, d\},\{a, c, d\},\{a, b, d\},\{a, b, c\},\{b, c, d\}$.

(viii) pre open sets in $(\mathrm{X}, \tau)$ are $\phi, X,\{a\},\{b\},\{a, b\},\{a, b, d\}$, $\{a, b, c\}$.

(ix) swg-open sets in $(\mathrm{X}, \tau)$ are $\phi, X,\{a\},\{b\},\{a, b\},\{a, b, c\}$, $\{a, b, d\}$

\subsection{Remark.}

From the above discussion and known results we have the following implications (Diagram 1).

$\mathrm{A} \rightarrow \mathrm{B}$ means A implies B but not conversely, A $\nrightarrow$ means A and $\mathrm{B}$ are independent.

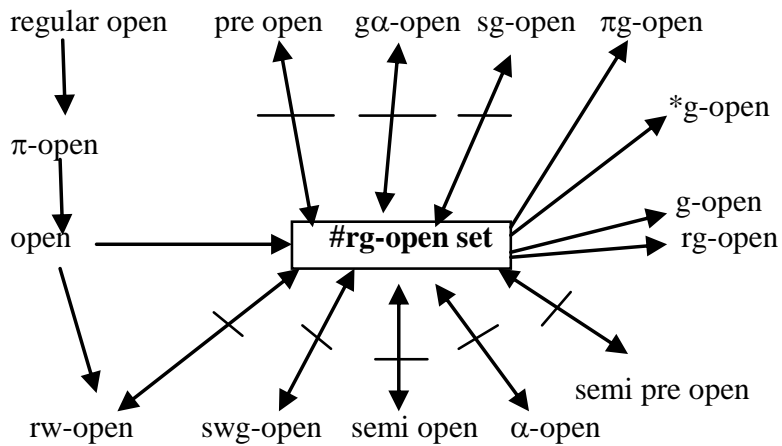

Diagram 1

\subsection{Theorem}

If $\mathrm{A}$ and $\mathrm{B}$ are \#rg-open set in a space $\mathrm{X}$. Then $\mathrm{A} \cap \mathrm{B}$ is also \#rg-open set in $\mathrm{X}$.

\section{Proof}

If $\mathrm{A}$ and $\mathrm{B}$ are $\#$ rg-open sets in a space $\mathrm{X}$. Then $\mathrm{X} \backslash \mathrm{A}$ and $\mathrm{X} \backslash \mathrm{A}$

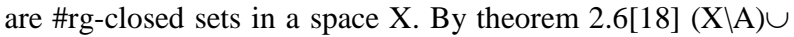
$(\mathrm{X} \backslash \mathrm{B})$ is also \#rg-closed sets in $\mathrm{X}$. Therefore $\mathrm{A} \cap \mathrm{B}$ is \#rgopen set in X.

\subsection{Remark}

The union of two \#rg-open sets in $\mathrm{X}$ is generally not a \#rgopen set in X.

\subsection{Example}

Let $X=\{a, b, c, d\}$ be with topology $\tau=\{X, \phi,\{a\},\{b\},\{a . b\}\}$ then the set $A=\{b, c\}$ and $B=\{b, d\}$ are \#rg-open set in $X$ but $A \cup B=\{b, c, d\}$ is not \#rg-open set in $X$

\subsection{Theorem}

If a subset $\mathrm{A}$ of a topological space $\mathrm{X}$ is both rw-closed and \#rg-open then it is open.

\section{Proof.}

Let $\mathrm{A}$ be rw-closed and \#rg-open set in $\mathrm{X}$. Now $\mathrm{A} \subseteq \mathrm{A}$. By

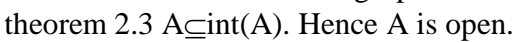

\subsection{Theorem}

If a set $\mathrm{A}$ is \#rg-open in $\mathrm{X}$, then $\mathrm{G}=\mathrm{X}$, whenever $\mathrm{G}$ is rw-open and $(\operatorname{int}(\mathrm{A}) \cup(\mathrm{X} \backslash \mathrm{A})) \subseteq \mathrm{G}$.

Proof

Suppose that $\mathrm{A}$ is \#rg-open in $\mathrm{X}$. Let $\mathrm{G}$ is rw-open and $(\operatorname{int}(\mathrm{A}) \cup(\mathrm{X} \backslash \mathrm{A})) \subseteq \mathrm{G}$. Thus $\mathrm{G}^{\mathrm{c}} \subseteq\left(\operatorname{int}(\mathrm{A}) \cup \mathrm{A}^{\mathrm{c}}\right)^{\mathrm{c}}=(\operatorname{int}(\mathrm{A}))^{\mathrm{c}} \cap \mathrm{A}$. That is $G^{c} \subseteq(\operatorname{int}(A))^{c} \backslash A^{c}$. Since $(\operatorname{int}(A))^{c}=\operatorname{cl}\left(A^{c}\right), G^{c} \subseteq \operatorname{cl}\left(A^{c}\right) \backslash$ $A^{c}$. Now, $G^{c}$ is rw-closed and $A^{c}$ is \#rg-closed, by theorem $2.7[18], \mathrm{G}^{\mathrm{c}}=\phi$. Hence $\mathrm{G}=\mathrm{X}$.

\subsection{Theorem}

Every singleton point set in a space is either \#rg-open (or) rwclosed.

\section{Proof}

It is follows from theorem $2.8[18]$.

\subsection{Definition}

Let $\mathrm{X}$ be a topological space and let $\mathrm{x} \in \mathrm{X}$. A subset $\mathrm{N}$ of $\mathrm{X}$ is said to be a \#rg-nbhd of $\mathrm{X}$ iff there exists a \#rg-open set $\mathrm{U}$ such that $\mathrm{x} \in \mathrm{U} \subseteq \mathrm{N}$.

\subsection{Definition}

A subset $\mathrm{N}$ of space $\mathrm{X}$, is called a \#rg-nbhd of $\mathrm{A} \subset \mathrm{X}$ iff there exists a \#rg-open set $\mathrm{U}$ such that $\mathrm{A} \subseteq \mathrm{U} \subseteq \mathrm{N}$.

\subsection{Theorem}

Every nbhd $\mathrm{N}$ of $\mathrm{x} \in \mathrm{X}$ is a \#rg-nbhd of $\mathrm{X}$, but not conversely.

\section{Proof}

Let $\mathrm{N}$ be a nbhd of point $\mathrm{x} \in \mathrm{X}$. Then there exists an open set $\mathrm{U}$ such that $\mathrm{x} \in \mathrm{U} \subseteq \mathrm{N}$. Since every open set is \#rg-open set, $\mathrm{U}$ is a \#rg-open set such that $\mathrm{x} \in \mathrm{U} \subseteq \mathrm{N}$. This implies $\mathrm{N}$ is \#rgnbhd of $\mathrm{x}$.

The converse of the above theorem need not be true as seen from the following example.

\subsection{Example}

Let $X=\{a, b, c, d\}$ with topology $\tau=\{X, \phi,\{a\},\{b\},\{a, b\}$, $\{a, b, c\}\}$. Then \#RGO $(X)=\{X, \phi,\{a\},\{b\},\{c\},\{a, b\},\{a, c\}$, $\{b, c\},\{a, b, c\}\}$. The set $\{c, d\}$ is \#rg-nbhd of the point $c$, since the \#rg-open sets $\{c\}$ is such that $c \in\{c\} \subset\{c, d\}$. However, the set $\{c, d\}$ is not a nbhd of the point $c$, since no open set $U$ exists such that $\mathrm{c} \in \mathrm{U} \subseteq\{\mathrm{c}, \mathrm{d}\}$.

\subsection{Theorem}

Every \#rg-open set is \#rg-nbhd of each of its points, but not conversely.

\section{Proof}

Suppose $\mathrm{N}$ is \#rg-open. Let $\mathrm{x} \in \mathrm{N}$. For $\mathrm{N}$ is a \#rg-open set such that $x \in N \subseteq N$. Since $x$ is an arbitrary point of $N$, it follows that $\mathrm{N}$ is a \#rg-nbhd of each of its points.

The converse of the above theorem is not true in general as seen from the following example.

\subsection{Example}

Let $X=\{a, b, c, d\}$ with topology $\tau=\{X, \phi,\{a\},\{b\},\{a, b\}\}$ Then \#RGO $(X)=\{X, \phi,\{a\},\{b\},\{c\},\{d\},\{a, b\},\{a, c\},\{b, c\}$, $\{\mathrm{a}, \mathrm{d}\},\{\mathrm{a}, \mathrm{c}\}\}$. The set $\{\mathrm{b}, \mathrm{d}\}$ is a \#rg-nbhd of the point $\mathrm{b}$, since the \#rg-open set $\{b\}$ is such that $c \in\{b\} \subseteq\{b, d\}$. Also the set $\{b, d\}$ is a \#rg-nbhd of the point $\{d\}$, Since the \#rgopen set $\{d\}$ is such that $d \in\{d\} \subseteq\{c, d\}$. Hence $\{b, d\}$ is a \#rg-nbhd of each of its points, but the set $\{b, d\}$ is not a \#rgopen set in X. 


\subsection{Remark}

The \#rg-nbhd $\mathrm{N}$ of $\mathrm{x} \in \mathrm{X}$ need not be a \#rg-open in $\mathrm{X}$. It is seen from the following example.

\subsection{Example}

Let $X=\{a, b, c, d\}$ with topology $\tau=\{X, \phi,\{a\},\{b\},\{c\}$, $\{a, b\},\{a, c\},\{b, c\},\{a, b, c\}\}$. Then $\# \operatorname{RGO}(X)=\{X, \phi,\{a\},\{b\},\{c\}$, $\{a, b\},\{a, c\},\{b, c\},\{a, b, c\}\}$. Here $\{a, b, d\}$ is a \#rg-nbhd of $\{a\}$, since $\{a, b\}$ is a \#rg-open set such that $a \in\{a, b\} \subseteq\{a, b, d\}$, but it is not a \#rg-open set.

\subsection{Theorem}

If $\mathrm{F}$ is a \#rg-closed subset of $\mathrm{X}$, and $\mathrm{x} \in \mathrm{F}^{\mathrm{c}}$ then there exists a \#rg-nbhd $\mathrm{N}$ of $\mathrm{x}$ such that $\mathrm{N} \cap \mathrm{F}=\phi$.

\section{Proof}

Let $\mathrm{F}$ be \#rg-closed subset of $\mathrm{X}$ and $\mathrm{x} \in \mathrm{F}^{\mathrm{c}}$. Then $\mathrm{F}^{\mathrm{c}}$ is \#rg-open set of $\mathrm{X}$. So by theorem 2.32. $\mathrm{F}^{\mathrm{c}}$ contains a \#rg-nbhd of each of its points. Hence there exists a \#rg-nbhd $\mathrm{N}$ of $\mathrm{x}$ such that $\mathrm{N} \subseteq \mathrm{F}^{\mathrm{c}}$. Hence $\mathrm{N} \cap \mathrm{F}=\phi$.

\section{3.\#RG-CLOSURE AND THEIR PROPERTIES.}

\subsection{Definition}

For a subset $\mathrm{A}$ of $\mathrm{X}, \# \operatorname{rg}-\mathrm{cl}(\mathrm{A})=\cap\{\mathrm{F}: \mathrm{A} \subseteq \mathrm{F}, \mathrm{F}$ is $\#$ rg closed in $\mathrm{X}\}$

\subsection{Definition}

Let $(\mathrm{X}, \tau)$ be a topological space and $\tau_{\text {\#rg }}=\{\mathrm{V} \subseteq \mathrm{X}$ : \#rg$\operatorname{cl}(\mathrm{X} \backslash \mathrm{V})=\mathrm{X} \backslash \mathrm{V}\}$.

\subsection{Definition}

For any $\mathrm{A} \subseteq \mathrm{X}, \# \operatorname{rg}$-int(A) is defined as the union of all \#rgopen set contained in A.

\subsection{Remark}

If $\mathrm{A} \subseteq \mathrm{X}$ is \# rg-closed then $\# \mathrm{rg}-\mathrm{cl}(\mathrm{A})=\mathrm{A}$, but the converse is not true.

\subsection{Example}

Let $X=\{a, b, c, d\}$ be with topology $\tau=\{X, \phi,\{a\},\{b\},\{a . b\}\}$. Let $A=\{a\}$ then $\# \operatorname{rg}-\operatorname{cl}(A)=A$, but $A$ is not \#rg-closed.

\subsection{Theorem}

Suppose $\tau_{\# \text { \#g }}$ is a topology. If $\mathrm{A}$ is \#rg-closed in $(\mathrm{X}, \tau)$, then $\mathrm{A}$ is closed in $\left(\mathrm{X}, \tau_{\# \mathrm{rg}}\right)$.

\section{Proof}

Since $\mathrm{A}$ is $\#$ rg-closed in $(\mathrm{X}, \tau)$, $\# \operatorname{rg}-\mathrm{cl}(\mathrm{A})=\mathrm{A}$. This implies $\mathrm{X} \backslash \mathrm{A} \in \tau_{\text {\#rg }}$. That is $\mathrm{X} \backslash \mathrm{A}$ is open in $\left(\mathrm{X}, \tau_{\text {\#rg }}\right)$. Hence $\mathrm{A}$ is closed in $\left(X, \tau_{\# \mathrm{rg}}\right)$

\subsection{Remark}

(i) $\quad \# \operatorname{rg}-\operatorname{cl}(\phi)=\phi$ and $\# \operatorname{rg}-\mathrm{cl}(\mathrm{X})=\mathrm{X}$

(ii) $\quad \mathrm{A} \subseteq \# \operatorname{rg}-\mathrm{cl}(\mathrm{A})$.

\subsection{Theorem}

For any $\mathrm{x} \in \mathrm{X}, \mathrm{x} \in \# \operatorname{rg}$-cl(A) if and only if $\mathrm{V} \cap \mathrm{A} \neq \phi$ for every $\#$ rg-open set $\mathrm{V}$ containing $\mathrm{x}$.

\section{Proof}

(Necessity). Suppose there exists a \#rg-open set $\mathrm{V}$ containing $\mathrm{x}$ such that $\mathrm{V} \cap \mathrm{A}=\phi$. Since $\mathrm{A} \subseteq \mathrm{X}|\mathrm{V}, \# \operatorname{rg}-\mathrm{cl}(\mathrm{A}) \subseteq \mathrm{X}| \mathrm{V}$ implies $\mathrm{x} \notin \#$ rg-cl(A) a contradiction.
(Suffiency).Suppose $\mathrm{x} \notin \#$ rg-cl(A), then there exists a \#rgclosed subset $F$ containing A such that $x \notin F$. Then $x \in X \mid F$ and $\mathrm{X} \mid \mathrm{F}$ is \#rg-open. Also $(\mathrm{X} \mid \mathrm{F}) \cap \mathrm{A}=\phi$, a contradiction.

\subsection{Remark}

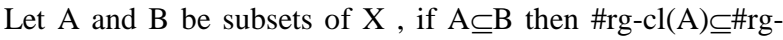
$\operatorname{cl}(\mathrm{B})$.

\subsection{Theorem}

Let $\mathrm{A}$ and $\mathrm{B}$ be subsets of $\mathrm{X}$, then $\# \operatorname{rg}-\mathrm{cl}(\mathrm{A} \cap \mathrm{B}) \subseteq$ $\#$ rg-cl(A) $\cap \#$ rg-cl(B).

\section{Proof}

Since $\mathrm{A} \cap \mathrm{B} \subseteq \mathrm{A}$ and $\mathrm{B}$, by remark 3.9, $\# \operatorname{rg}-\mathrm{cl}(\mathrm{A} \cap \mathrm{B}) \subseteq \# \operatorname{rg}-\mathrm{cl}(\mathrm{A})$ and, $\# \operatorname{rg}-\mathrm{cl}(\mathrm{A} \cap \mathrm{B}) \subseteq \# \operatorname{rg}-\mathrm{cl}(\mathrm{B})$.Thus, $\# \operatorname{rg}-\mathrm{cl}(\mathrm{A} \cap \mathrm{B}) \subseteq \# \operatorname{rg}-\mathrm{cl}(\mathrm{A})$

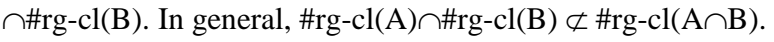

\subsection{Example}

Let $X=\{a, b, c, d\}$ be with topology $\tau=\{X, \phi,\{a\},\{b\},\{a . b\}$, $\{a \cdot b, c\}\}$. Let $A=\{a\}$ and $B=\{b\}$ and $\# \operatorname{rg}-c l(A)=\{a, d\}$, \#rg $\operatorname{cl}(B)=\{b, d\}$. Then $\# \operatorname{rg}-c l(A) \cap \# \operatorname{rg}-c l(B)=\{d\} \not \subset \# \operatorname{rg}-c l(A \cap B)$.

\subsection{Theorem}

If $A$ and $B$ are \#rg-closed sets then \# $\operatorname{rg}-c l(A \cup B)=$ $\#$ rg-cl(A) $\cup$ Hrg-cl(B).

\section{Proof}

Let $\mathrm{A}$ and $\mathrm{B}$ be \# rg-closed in $\mathrm{X}$. Then $\mathrm{A} \cup \mathrm{B}$ is also \#rgclosed. Then \#rg-cl $(A \cup B)=A \cup B=\# \operatorname{rg}-c l(A) \cup \# r g-c l(B)$.

\subsection{Theorem}

$(\mathrm{X} \backslash \# \operatorname{rg}$-int $(\mathrm{A}))=\# \operatorname{rg}-\mathrm{cl}(\mathrm{X} \backslash \mathrm{A})$

\section{Proof}

Let $\mathrm{x} \in \mathrm{X} \backslash \#$ rg-int(A), then $\mathrm{x} \notin \#$ rg-int(A). Thus every \#rgopen set $\mathrm{B}$ containing $\mathrm{x}$ is such that $\mathrm{B} \not \subset \mathrm{A}$. This implies every \#rg-open set $B$ containing $x$ intersects $X \backslash A$. This means

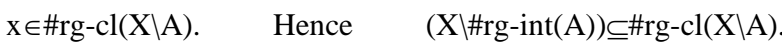
Conversely, let $\mathrm{x} \in \#$ rg-cl(X\A). Then every \#rg-open set $\mathrm{U}$ containing $\mathrm{x}$ intersects $\mathrm{X} \backslash \mathrm{A}$. That is every \#rg-open set $\mathrm{U}$ containing $\mathrm{x}$ is such that $\mathrm{U} \not \subset \mathrm{A}$, implies $\mathrm{x} \notin \#$ rg-int(A). Hence $\# \operatorname{rg}-\mathrm{cl}(\mathrm{X} \backslash \mathrm{A}) \subseteq(\mathrm{X} \backslash \# \operatorname{rg}-\operatorname{int}(\mathrm{A}))$. Thus $\quad(\mathrm{X} \backslash \# \operatorname{rg}-\operatorname{int}(\mathrm{A}))=\#$ rg$\operatorname{cl}(\mathrm{X} \backslash \mathrm{A})$.

\section{CONCLUSION}

In this paper, we have introduced the weak and generalized form of open sets namely \#rg-open set and established their relationships with some generalized sets in topological space.

\section{REFERENCES}

[1] Benchalli. S.S.,and Wali. R.S.,On RW-Closed sets in topological spaces, Bull. Malays. Math. Sci. Soc(2) 30(2) (2007), $99-110$

[2] Bhattacharyya. P. and Lahiri.B.K., Semi-generalized closed sets in topology, Indian J. Math. 29(1987), 376382 . 
[3] Biswas. N., On characterization of semi-continuous functions, Atti Accad. Naz. Lincei Rend, Cl. Sci. Fis. Mat. Natur. 48(8)(1970), 399-402.

[4] Cameron. D.E., Properties of S-closed spaces, Proc. Amer Math. Soc. 72(1978), 581-586.

[5] Dontchev. J. and Noiri.T., Quasi-normal spaces and $\pi \mathrm{g}$ closed sets, Acta Math. Hungar. 89(3)(2000), 211-219.

[6] Gnanambal. Y., On generalized preregular closed sets in topological spaces, Indian J. Pure App. Math. 28(1997), 351-360.

[7] Gnanambal.Y. and Balachandran.K., On gpr-continuous functions in topological spaces, Indian J. Pure Appl. Math. 30(6)(1999), 581-593.

[8] Levine. N., Semi-open sets and semi-continuity in topological spaces, Amer. Math. Monthly,70(1963), 3641

.[9] Levine. N., Generalized closed sets in topology, Rend. Circ. Mat. Palermo 19(1970), 89-96.

[10] Maki. H, Devi. R and Balachandran. K., Associated topologies of generalized $\alpha$-closed sets and $\alpha$ generalized closed sets, Mem. Sci. Kochi Univ. Ser. A. Math. 15(1994), 51-63.

[11] Mashhour. A.S., Abd. El-Monsef. M. E. and El-Deeb S.N., On pre continuous mappings and weak precontinuous mappings, Proc Math, Phys. Soc. Egypt 53(1982), 47-53.
[12] Nagaveni. N., Studies on Generalizations of Homeomorphisms in Topological Spaces, Ph.D. Thesis, Bharathiar University, Coimbatore, 1999

. [13] Palaniappan. N., and Rao. K. C., Regular generalized closed sets, Kyungpook Math. J. 33(1993), 211-219.

[14] Park. J. K. and Park. J.H., Mildly generalized closed sets, almost normal and mildly normal spaces, Chaos, Solitions and Fractals 20(2004), 1103-1111.

[15] Pushpalatha. A., Studies on Generalizations of Mappings in Topological Spaces, Ph.D. Thesis, Bharathiar University, Coimbatore, 2000.

[16] Stone. M., Application of the theory of Boolean rings to general topology, Trans. Amer. Math. Soc. 41(1937), 374-481.

[17] Sundaram.P and Sheik John.M., On w-closed sets in topology, Acta Ciencia Indica 4(2000), 389-392

[18] Syed Ali Fathima. S and Mariasingam. M, On \#regular generalized closed sets in topological spaces, International journal of mathematical archive-2(11), $2011,2497-2502$

[19] Veera Kumar M.K.R.S., Between g* closed sets and gclosed sets , Mem.Fac.Sci.Kochi Univ. Ser .App .Math ,21 (2000),1-19.

[20] Zaitsav V(1968) On certain classes of topological spaces and their bicompactifications. Dokl. Akad. Nauk SSSR 178: $778-779$ 